\title{
RESISTENCIA ANTIMICROBIANA DE Staphylococcus AISLADOS DE LA PIEL DE GATOS. ¿UN RIESGO PARA LA SALUD HUMANA?
}

Sonia Anticevic C, María Antonieta Jara O, Loreto Muñoz A.

Financiado por Proyecto FIV 121014019102008

Actualmente, las patologías dermatológicas son una de las causas de consulta más frecuentes en la práctica veterinaria de animales pequeños, posiblemente debido a los evidentes signos y lesiones que presentan los animales: prurito, alopecia y olor desagradable, entre otros.

Esta alta frecuencia de consultas dermatológicas se ha evidenciado en diversos países. Así, en un estudio realizado por Hill, et al., en 2006, se concluyó que las patologías dermatológicas de animales pequeños son la segunda causa de consulta en las clínicas veterinarias en Edimburgo (13\%). Los signos clínicos dermatológicos de los gatos estudiados fueron inflamación cutánea (36\%), prurito y otitis (19\%) y alopecia (15\%) y por categorías de diagnóstico de las patologías cutáneas, los parásitos e infecciones bacterianas ocuparon el primer lugar. Un escenario similar fue revelado por un estudio realizado en el Hospital Clínico Veterinario de la Universidad de Chile entre los años 2002 y 2005, en donde se atendieron un total de 2.063 gatos, evidenciando que $279(13,5 \%)$ acudieron a consulta por patologías dermatológicas (Rossel, 2009)

Las bacterias del género Staphylococcus son microorganismos residentes de la microflora normal de las mucosas y piel de humanos y animales (Scott, et al., 2001; Lee, et al., 2003). Sin embargo, algunas especies son patógenos oportunistas que pueden causar serias enfermedades cutáneas en tejidos o cavidades (Scott, et al., 2001). La transmisión de estas bacterias puede ser por contacto directo de piel con piel, por aerosoles de estornudos y tos y también a través de la saliva (Malik,et al., 2007). 
Dentro del género Staphylococcus, se describe a S. aureus como una bacteria comensal en humanos y patógeno en animales pequeños (perros y gatos), estando poco estudiada su prevalencia y los potenciales sitios de colonización en ellos (Scott, 2005). Los humanos son portadores de S. aureus, describiéndose que entre el 25 a 38\% de la población la portan en la cavidad nasal (Scott, 2005; Simou et al., 2005; Boost et al., 2008; Acton, et al., 2009) Con respecto a los gatos, Se ha documentado que los animales sanos lo transportan en su nariz (Cox et al., 1985; Abraham, et al., 2007) y también puede encontrarse en lesiones de piel (Devriese, et al., 1984; Medleau y Blue, 1988; Lilenbaum, et al., 1998). Algunos investigadores sugieren que los aislamientos de Staphylococcus aureus a partir del gato es debido a la transmisión de esta bacteria desde su propietario (Devriese et al.,1984; Cox et al., 1985) Los Staphylococcus generalmente no son causantes de enfermedades específicas de piel en gatos, sin embargo, se ha descrito su participación como agente contaminante y perpetuante de las dermatitis superficiales, foliculitis bacteriana y pioderma superficial, siendo posible también encontrarlo como agente causal de las patologías mencionadas (Malik, et al., 2007; Wildermuth, et al., 2006).

Por otro lado, S. intermedius es una bacteria comensal en felinos, que habita normalmente en sitios anatómicos como nariz, labios, faringe, conjuntiva ocular, pabellón auricular, región perianal, vagina y prepucio (Cox, et al., 1985; Lilenbaum, et al., 1998). Otros Staphylococcus coagulasa (+) como S. hyicus y varios coagulasa (-) como $S$. felis, también han sido aislados de piel y mucosas de gatos sanos (Cox, et al., 1985; Igimi, et al., 1994; Lilenbaum, et al., 1998; Patel, et al., 2002; Baptiste y Williams 2005).

Existen numerosos estudios disponibles en la literatura que señalan el aislamiento de Staphylococcus coagulasa positivos y coagulasa negativos desde gatos enfermos y sanos: Así, Lilenbaum et al., (1998) aislaron Staphylococcus coagulasa positivos en muestras obtenidas de 98 gatos clínicamente sanos, siendo las especies más frecuentemente aisladas $S$. intermedius $(26,5 \%)$ y $S$. aureus $(14,3 \%)$. 
Lilenbaum et al., (1999) estudiaron la flora bacteriana aeróbica residente en la saliva de 104 gatos clínicamente sanos, aislando 30 cepas $(28,8 \%)$ de $S$. Intermedius, siendo la segunda especie más frecuentemente aislada y la única coagulasa positiva. Patel et al., (1999) obtuvieron muestras de felinos domésticos sanos, con lesiones y felinos silvestres $(n=30)$, obteniendo 187 aislados de Staphylococcus, de los cuales el 21,4\% correspondieron a especies coagulasa positivas, siendo $S$. intermedius la que se aisló con mayor frecuencia (90\%), correspondiendo el resto de los aislados a S. aureus (10\%).

Morris, et al., (2006a) en un total de 11 gatos, aislaron como agente primario a $S$. aureus meticilino resistente (MRSA) en gatos con obstrucción uretral, neoplasia y colangiohepatitis; en cambio se aisló como agente secundario S.aureus meticilino sensible (MSSA) en 27 gatos que cursaban con enfermedades de piel y oído, tracto genitourinario, neoplasia y enfermedades cardiovasculares. Por último, Abraham et al., (2007) estudiaron la presencia de S. aureus, S. intermedius y $S$. schleiferi en piel y mucosas de gatos sanos y con enfermedad inflamatoria de piel, obteniendo aislamientos positivos en el $50 \%$ de los animales enfermos y en el $34 \%$ de animales sanos. S.aureus obtuvo una frecuencia de aislamiento de $59 \%$ y $58 \%$ en gatos sanos y enfermos, respectivamente. S.intermedius fue aislado en el $65 \%$ y $46 \%$ de gatos sanos y enfermos, respectivamente.

Staphylococcus schleiferi fue descrito por primera por Igimi et al. (1990), a partir de una otitis externa en un perro. Esta especie puede ser coagulasa positivo o negativo, siendo difícil diferenciarlo del Staphylococcus aureus mediante las pruebas convencionales. Además, en numerosos reportes no se indicaba la subespecie debido a que con las pruebas bioquímicas tradicionales o con algunos kit comerciales no lograban diferenciar los coagulasa positivo (subsp. coagulans) de los negativo (subsp. schleiferi), es así como actualmente los laboratorios comerciales incluyeron en sus paneles pruebas bioquímicas que logran hacer la diferenciación entre ellos (Kumar et al., 2007).

S. schleiferi fue aislado e identificado, por primera vez en Chile, en piodermas y otitis de perros. (Muñoz et al., 2008) Además, fue aislado por primera vez en Chile de gatos sanos y con lesiones dermatológicas en el 
Laboratorio de Microbiología de la Facultad de Ciencias Veterinarias, de la Universidad de Chile, a partir de muestras obtenidas de pacientes atendidos en el Hospital de la Facultad. (Sepúlveda et al., 2010)

En alguna etapa de su vida, perros y gatos sufren enfermedades cutáneas e infecciones por Staphylococcus como pioderma y otitis externa, las que son tratadas con antimicrobianos. (Fotografías $N^{0} 1$ y 2) Esta práctica ha permitido el desarrollo de resistencia bacteriana en aislados de Staphylococcus. Los estudios de susceptibilidad antimicrobiana en animales de compañía han revelado que cepas de Staphylococcus aisladas de gatos y perros exhiben patrones de resistencia similares a los de esta bacteria en humanos y que el desarrollo de esta resistencia está influido por la alta frecuencia de uso de algunos antimicrobianos (Malik et al., 2007).

En medicina veterinaria, la obtención de una muestra para estudio de cultivo y antibiograma no es considerada una práctica habitual cuando se sospecha que el pioderma u otitis externa tiene como agente involucrado Staphylococcus, utilizándose antimicrobianos con efectividad predecible sobre S. intermedius. Por lo anterior, sólo los casos refractarios a la terapia antimicrobiana sistémica son sometidos a estudios de susceptibilidad (Morris et al., 2006b).

Dentro de los antimicrobianos utilizados en la terapia empírica de dermatopatías causadas por Staphylococcus se encuentran penicilinas, cefalosporinas, macrólidos, lincosamidas, tetraciclinas, cloranfenicol, sulfonamidas potenciadas, aminoglucósidos y fluoroquinolonas (Watson y Rosin, 2000). Años más tarde, Hill et al., (2006) señalaron que en Escocia, entre los años 1998 y 2001, los antimicrobianos más utilizados en el tratamiento de patologías dermatológicas fueron cefalexina, amoxicilina, amoxicilina con ácido clavulánico y enrofloxacino.

Analizando datos internacionales, Heuer et al., (2005) encontró que en Dinamarca el incremento del uso de antimicrobianos en las mascotas, entre los años 2001 y 2004, fue de un $70 \%$, en base a las cefalosporinas, por lo cual 
advierte que puede aumentar la resistencia antimicrobiana, la que se puede traspasar a los humanos debido al estrecho contacto con las mascotas.

Desde hace ya varios años, se ha observado resistencia bacteriana a los antimicrobianos mencionados, teniendo como consecuencia fracasos terapéuticos y perpetuación del estado de enfermedad en los pacientes. La resistencia bacteriana es actualmente un tema de gran preocupación mundial en Medicina Humana y Veterinaria (Sanders, 1999; Codex Alimentarius, 2000; Organización Mundial de la Salud, 2000; Denamiel et al., 2009). Las investigaciones, tanto clínicas como epidemiológicas, han demostrado que cada vez son menos las barreras para el paso de genes de resistencia entre diferentes poblaciones bacterianas, incluida la transferencia desde bacterias comensales a patógenas, la transferencia de bacterias patógenas y no patógenas de los animales al hombre y al medio ambiente (Davison et al., 2000; Anderson et al., 2008; Umber y Bender, 2009).

Los primeros estudios se enfocaron a reportar e identificar Staphylococcus spp aislados de piel y mucosas de gatos sanos y gatos con enfermedades a la piel estudiando la susceptibilidad antimicrobiana, con lo cual se puede evidenciar que, a través de los años, se ha incrementado el nivel de resistencia de estas bacterias a los antimicrobianos.

Así, en el año 1988, Medleau y Blue, encontraron que el $100 \%$ de los S.aureus $(n=16)$ presentaron sensibilidad a cloxacilina, cloranfenicol, gentamicina y eritromicina, mientras un $50 \%$ fueron sensibles a la penicilina $G$ y ampicilina. Años más tarde, Lilenbaum et al., (1998) encontró mayores porcentajes de resistencia a gentamicina $(5,1 \%)$ y a cloxacilina $(22,5 \%)$. Morris et al., (2006a) encontró una diferencia significativa de la resistencia antimicrobiana, en donde las cepas de $S$. aureus resistentes a meticilina (MRSA) presentaron $100 \%$ de resistencia a la fluoroquinolonas y macrólidos; en cambio los $S$. aureus sensibles a meticilina (MSSA) mantuvieron frecuencias altas de susceptibilidad, con lo que se demostró que las cepas al ser meticilino-resistente marcan una tendencia de resistencia hacia otros antimicrobianos. Patel et al., (1999) encontró mayor resistencia antimicrobiana en los aislados bacterianos de MSSA de gatos salvajes en comparación a los gatos con dueños, esto debido a que los gatos salvajes están más expuestos a 
la contaminación ambiental de la ciudad, posiblemente originada por hospitales y servicios de la salud humana.

Así Lloyd (2007), reportó que la resistencia antimicrobiana del Staphylococcus intermedius, principal agente del pioderma canino y felino, se ha incrementado con los años, incluso apareciendo bacterias con multirresistencia (resistencia a más de 3 antimicrobianos). Morris et al., (2006a) encontró una diferencia significativa de la resistencia antimicrobiana, en donde las cepas MRSA presentaron $100 \%$ de resistencia a la fluoroquinolonas y macrólidos; en cambio los $S$. aureus sensibles a meticilina (MSSA) mantuvieron frecuencias altas de susceptibilidad, con lo que se demostró que las cepas al ser meticilino-resistente marcan una tendencia de resistencia hacia otros antimicrobianos.

La presencia de diferentes especies bacterianas en la flora normal de pequeños animales, determina que perros y gatos sean considerados como reservorios de estas bacterias y de genes de resistencia que tienen importancia clínica en humanos como MRSA, Enterococcus resistente a vancomicina y Salmomella Typhimurium DT104 multirresistente (Guardabassi et al., 2004).

La mayor existencia de animales de compañía que son potenciales portadores de bacterias con determinantes de resistencia, son un riesgo para los propietarios, especialmente si éstos tienen una mayor susceptibilidad a infecciones. Se ha reportado que las mascotas se comportan como reservorios de bacterias resistentes, como MRSA, al ser los perros y gatos contaminados por sus propietarios (Guardabassi et al., 2004; Morgan, 2008). Se ha documentado que los humanos adquieren los MRSA mayoritariamente de la exposición directa a ambientes hospitalarios y éste lo transmite a su mascota, produciéndole a ella en algunos casos patologías o bien lo mantienen como portador nasal (Duquette y Nuttall, 2004; Vitale, et al., 2006).

Strommenger et al., (2006) concluye con su estudio que los aislados de MRSA de las mascotas, son originarios de clones de hospitales regionales; o 
como indica Weese et al. (2005) clones predominantes de la comunidad de un lugar geográfico determinado.

En un estudio realizado por Morris et al. (2010), demostraron que los humanos y sus mascotas pueden ser portadores de cepas idénticas de estafilococos resistentes a meticilina (MR) cuando comparten el mismo ambiente. En la investigación participaron veterinarios especialistas en dermatología y empleados de las clínicas dermatológicas, ambos grupos con una elevada exposición a animales infectados con Staphylococcus spp. El objetivo de este fue evaluar la prevalencia de portadores de MR Staphylococcus aureus (MRSA), MR S. pseudintermedius (MRSP) y MR S. schleiferi (MRSS) entre veterinarios, empleados de las clínicas y sus mascotas personales. Participaron 171 personas y sus mascotas (258 perros y 160 gatos). Las muestras de las 171 personas dieron un total de seis aislados de MRSA (3.5\%), nueve de MRSP (5.3\%) y cuatro de MRSS (2.3\%), mientras que las 418 mascotas presentaron un total de ocho aislados de MRSA (1.9\%), 21 de MRSP (5\%) y dos de MRSS (0.5\%). Las cepas idénticas (con igualdad genética mediante electroforesis de campo pulsado) fueron aisladas en cuatro de los 171 hogares: MRSA de una persona/2 mascotas y MRSP de tres personas/tres mascotas.

MRSA es uno de los problemas preocupantes en medicina humana, siendo una causa importante de muerte en diversos países (Guardabais, 2008); las mascotas pueden actuar como portadores sanos de estas bacterias (Morris et al., 2006b; Strommenger et al., 2006; Boost et al. 2008) pudiendo hacer la patología cuando se infectan heridas por mordeduras o postquirúrgicas; reportándose que los veterinarios tendrían mayor riesgo a contraer y portarlas (Guardabassi, 2008).

Podemos concluir entonces que, actualmente, el problema de la resistencia a los antibióticos es un hecho en las poblaciones animales que están en contacto continuo con el hombre. Es necesario entonces, implementar las medidas de vigilancia epidemiológica urgente de la situación de resistencia a antibióticos y una intensificación de las normas de uso prudente de los mismos. 


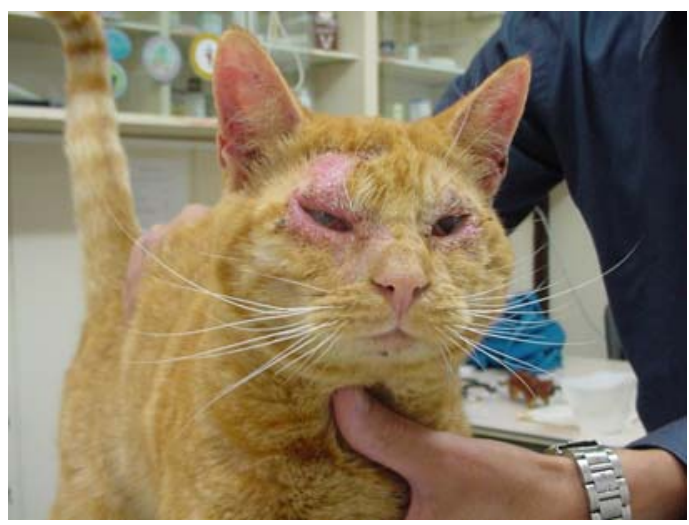

Fotografía №1: Paciente atendido en el Hospital Clínico Veterinario de la Universidad de Chile. Presenta eritema,

prurito de cabeza y cuello, contaminado con

Staphylococcus coagulasa positiva.

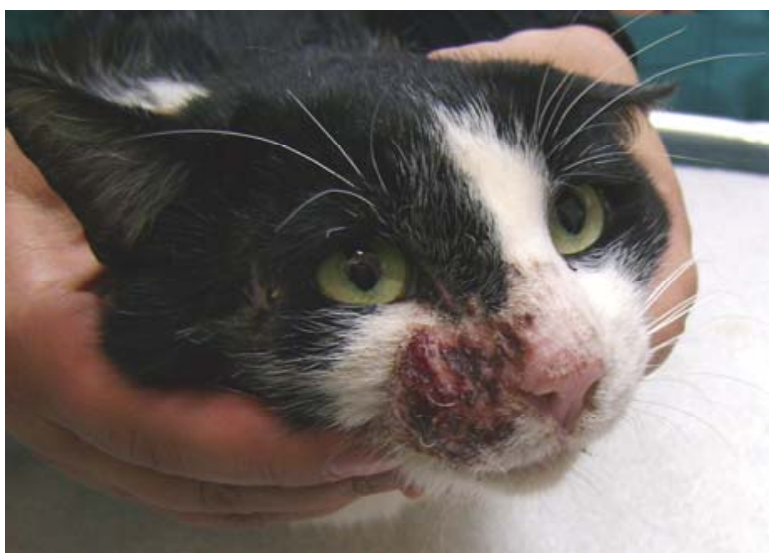

Fotografía №2: Paciente atendido en el Hospital Clínico Veterinario de la Universidad de Chile.

Presenta prurito de cabeza y cuello, granuloma eosinofílico contaminado con Staphylococcus coagulasa positiva.

\section{BIBLIOGRAFÍA}

ABRAHAM, J.; MORRIS, D.; GRIFFETH, G.;SHOFER, F.; RANKIN S. 2007 Surveillance of healthy cats and cats with inflammatory skin disease for colonization of the skin by methicillin resistant coagulase-positive staphylococci and Staphylococcus schleiferi ssp. schleiferi Vet. Dermatol. 18: 252-259.

ACTON, D.; TEMPELMANS PLAT-SINNIGE, M.; VAN WAMEL, W.; DE GROOT, N.;VAN BELKUM,A. 2009 Intestinal carriage of Staphylococcus aureus:how does its freuency compare with that of nasal carriage and what is its clinical impact? Eur J Clin Microbiol Infect Dis 28: 115 - 127

ANDERSON, M., LEFEBVRE S., WEESE S. 2008 Evaluation of prevalence and risk factors for methicillin-resistant Staphylococus aureus colonization in veterinary personnel attending an international equine veterinary conference. Vet Microbiol 129:410-417 
BAPTISTE,KE.; WILLIAMS, NJ. 2005 Methicillin resistant staphylococci in companion animals. Emerg. Infec. Dis.11:1942-4.

BOOST, M.; O'DONOGHUE, M.; JAMES, A. 2008. Prevalence of Staphylococcus aureus carriage among dogs and their owners. Epidemiol Infect 136:953-964

COMISIÓN DEL CODEX ALIMENTARIUS 2000 Programa conjunto FAO/OMS sobre normas alimentarias. $12^{\mathrm{a}}$ Reunión del Comité del Codex sobre Residuos de Medicamentos Veterinarios en los Alimentos. Apéndice B:1-13 .

COX,H.; HOSKINS J.; NEUMAN,S.; TURWALD,G.; FOIL,C.; ROY,A.; KEARNEY,M. 1985 Distribution of Staphylococcal species on clinically healthy cats. Am J Vet Res 46(9): 1824- 1828.

DAVISON, H.;LOW, J.; WOOLHOUSE, M. 2000 What is antibiotic resistance and how can we measure it? Trends in Microbiology 8:554-559.

DENAMIEL G, PUIGDEVALL T, MÁS J, ALBARELlOS G, GENTILINI E. Prevalencia y perfil de resistencia a betalactámicos en estafilococos de perros y gatos. InVet. 2009 Dic [citado 2011 Ene 10] ; 11(2): 117-122.Disponible en: http://www.scielo.org.ar/scielo

DEVRIESE, L.; NZUAMBE,D.; GODARD, C. 1984 Identification and characterization of Staphylococci isolated from cats. Vet Microbiol 9: 279-285.

DUQUETTE, R Y NUTTALL, T. 2004 Methicillin resistant staphylococcus aureus in dogs and cats: an emerging problem? J Small Anim Pract 45:591 _ 597.

GUARDABASSI, L., SCHWARZ, S., LLOYD, D. 2004 Review. Pet animal as reservoirs of antimicrobial-resistant bacteria. J.Antimicrob.Chemother. 54: 321 332. 
GUARDABASSI, L. 2008 Pet animals as reservoirs of antimicrobial-resistant bacteria: recente discoveries potentially impacting human health. $59^{\circ}$ Congresso Internazionale Multisala SCIVAC 30Mayo- 1Junio Rimini, Italia. 247-248

HEUER, O.; JENSEN, V.; HAMMERUM, A. 2005 Antimicrobial drug consumption in companion animals. Emerg Infect Dis 11:344-345

HILL, P.;EDEN, C.; HUNTLEY, S.; MOREY, V.; RAMSEY, S.; RICHARDSON, C.; SMITH, D.; SUTTON, C.; TAYLOR, M.; THORPE, E.; TIDMARSH, R.; WILLIAMS, V. 2006 Survey of the prevalence, diagnosis and treatment of dermatological conditions in small animals in general practice. Vet Rec 158: 533-539.

IGIMI, S.; TAKAHASHI, E.; MITSUOKA,T. 1990 Staphylococcus schleiferi subsp.coagulans subsp.nov., isolated from the external auditory meatus of dogs with external ear otitis. International Journal of Systematic Bacteriology 40: 409-411

IGIMI, S.; ATOBE,H.; TOHYA, Y.; INOUE,A.; TAKAHASHI,E.; KONISHI,S. 1994 Characterization of the most frequently encoutered Staphylococcus sp in cats. Vet Microbiol 39: 255- 260.

KUMAR, D.; CAWLEY, J.; IRIZARRY-ALVARADO, J.; ALVAREZ, A.; ALVAREZ, S. 2007 Case of Staphylococcus schleiferi subspecies coagulans endocarditis and metastatic infection in an immune compromised host. Transpl Infect Dis 9:336-338

LEE, P.K.; ZIPOLI M.Y.; WEINBERG A.N. 2003 Pyodermas: Staphylococcus aureus, Streptococcus and other Gram-positive bacteria. In Freedberg, IM.; Eisen, AZ.; Wolff, K. Eds. Fitzpatrick's Dermatology in general Medicine, 6th New York; McGraw-Hill Medical Publishing 1856- 78.

LILENBAUM, W.; NUNES, E.; AZEREDO,M. 1998 Prevalence and antimicrobial susceptibility of staphylococci isolated from the skin surface of clinically normal cats. Lett Appl Microbiol 27: $224-228$. 
LILENBAUM, W.; ESTEVES, A.L.; SOUZA, G.N. 1999 Prevalence and antimicrobial susceptibility of staphylococci isolated from saliva of clinically normal cats. Lett Appl Microbiol 28: 448-452.

LLOYD, D. 2007 Reservoirs of antimicrobial resistance in pet animals. Clin Infect Dis 45: 148-152

MALIK, S. CHISTENSEN, H.; PENG,H.; BARTON,M. 2007 Presence and diversity of the $\beta$ - lactamase gene in cat and dog staphylococci. Vet Microbiol $123(1-3)$ 162-168.

MEDLEAU, L., BLUE, L. 1988. Frequency and antimicrobial susceptibilityu of Staphylococcus spp isolated from feline skin lesions. JAVMA 193 (9): 1080 1081.

MORGAN, M. 2008 Methicillin - resistant Staphylococcus aureus and animals: zoonosis or humanosis? J Antimicrob Chemother 62:1181 -1187

MORRIS, D.; BOSTON, R.; O'SHEA, K.; RANKIN, S. 2010 The prevalence of carriage of meticillin-resistant staphylococci by veterinary dermatology practice staff and their respective pets. Vet Dermatol 21:400-407

MORRIS, D.; MAULDIN,E.;O'SHEA, K.; SHOFER, F.; RANKIN, S. $2006 a$. Clinical, microbiological, and molecular characterization of methicillin-resistant Staphylococcus aureus infections of cats. AJVR 67:1421-1425.

MORRIS, D.; ROOK, K.; SHOFER, F.; RANKIN, S. 2006b. Screening of Staphylococcus aureus, Staphylococcus intermedius and Staphylococcus schleiferi isolates obtained from small companion animals for antimicrobial resistance: a retrospective review of 749 isolates (2003-04) Vet. Dermt. 17: 332-337.

MUÑOZ, L.; ANTICEVIC, S.; BORIS, C.; SILVA, V.; ULLOA MT.; HERESSMAN, M.; ABUSLEME, F.; MOLINA, M. 2008. Primer aislamiento y susceptibilidad antimicrobiana de Staphylococcus schleiferi desde piodermas y 
otitis canina en Chile. XV Congreso Nacional de Medicina Veterinaria. 20 al 22 de Noviembre. Pucón, Temuco.

ORGANIZACIÓN MUNDIAL DE LA SALUD. 2000. Overcoming antimicrobial resistance. World Health Organization (Report on Infectious Diseases) Geneva, Switzerland p.67.

PATEL, A.; LLOYD,D.; LAMPORT, A. 1999. Antimicrobial resistance of feline staphylococci in South-eastern England. Vet Dermatol 10: 257 - 261.

PATEL,A.; LLOYD,H.; HOWELL,S.; NOBLE,W. 2002. Investigation into the potential pathogenicity of Staphylococcus felis in a cat. Vet Rec 150:668 669.

ROSSEL, D. 2009. Estudio descriptivo de afecciones dermatológicas en pacientes felinos. Memoria para optar al Título Profesional de Médico Veterinario. Fac. de Cs Vet y Pec, Universidad de Chile .

SANDERS, P. 1999. Eureopean Symposium. Antibiotic resistance in bacteria of animal origin. 29-30 November. Institut Pasteur- CIS. Paris Francia.

SEPÚLVEDA, A.; ANTICEVIC, S.; LUBI, P.; MUÑOZ, L. 2010 Determinación de susceptibilidad antimicrobiana en cepas de Staphylococcus coagulasa positivas aisladas desde gatos. XVI Congreso Nacional de Medicina Veterinaria, 18 al 20 de Noviembre, Concepción.

SIMOU, C.; HILL, P.; FORSYTHE, P.; THODAY, K. 2005. Species specificity in the adherence of staphylococci to canine and human corneocytes: a preliminary study. Vet Dermatol 16:156-161

SCOTT, D.W.; MILLER W.H.; GRIFFIN C.E. 2001. Bacterial skin diseases. In: Muller and Kirk's Small Animal Dermatology, 6th Edn. Philadelphia, PA:W.B.Saunders $274-335$.

SCOTT, J. 2005. Methicillin-resistant Staphylococcus aureus: An emerging pathogen in small animals. J Am Anim Hosp Assoc 41:150-157. 
STROMMENGER, B.; KEHRENBERG, C.; KETTLITZ, C.; CUNY, C.; VERPOHL, J.; WITTE, W.; SCHWARZ, S. 2006 Molecular characterization of methicillin-resistant Staphylococcus aureus strains from pet animals and their relationship to human isolates $\mathrm{J}$ Antimicrob Chemother 57:461 -465

UMBER, J.; BENDER, J. 2009 Pets and antimicrobial resistance. Vet Clin Small Anim 39:279 -292

VITALE, C.; GROSS,T.; WEESE,S. 2006. Methicillin resistant Staphylococcus aureus in cat and owner. Emerg Infect Dis 12(12):1998-1999.

WATSON, A.; ROSIN, E. 2000. Antimicrobial drug use in dogs and cats. In Antimicrobial Therapy in Veterinary Medicine, $3^{\text {rd }}$ Edn Prescott, J.F, Baggot; J.D. and Walker R.D. pp. 537-575. USA:Iowa State University Press.

WEESE, J.S. 2005. Methicillin-Resistant Staphylococcus aureus: An Emerging Pathogen in Small Animals. J. of the Amer. Anim. Hospt. Associa. 41: 150157.

WEESE, J.; DICK, H.; WILLEY, B.; MCGEER,A.; KREISWIRTH,B.; INNIS,B.; LOW,D. 2006 Suspected transmission of methicillin-resistant Staphylococcus aureus between domestic pets and hmans in veterinary clinics and in the household. Vet Microbiol 115: 148 -155

WILDERMUTH, B.; GRIFFING, C.; ROSENKRANTZ, W. 2006. Feline pyoderma therapy. Clin Tech Small Anim Pract 21:150-156.

ZDOVC, I., OCEPEK, M., PIRS, T., KRT, B.; PINTER, L. 2004 Microbiological features of Staphylococcus schleiferi subsp. coagulans, isolated from dogs and possible misidentification with other canine coagulase- positive Staphylococci. J Vet Med 51: 449-454 
